\title{
VANISHING PROPERTIES OF DUAL BASS NUMBERS
}

\author{
LINGGUANG LI
}

\begin{abstract}
Let $R$ be a Noetherian ring, $M$ an Artinian $R$-module, $\mathfrak{p} \in \operatorname{Cos}_{R} M$. Then $\operatorname{cograde}_{R_{\mathfrak{p}}} \operatorname{Hom}_{R}\left(R_{\mathfrak{p}}, M\right)=\inf \left\{i \mid \pi_{i}(\mathfrak{p}, M)>0\right\}$ and $\pi_{i}(\mathfrak{p}, M)>0 \Rightarrow \operatorname{cograde}_{R_{\mathfrak{p}}} \operatorname{Hom}_{R}\left(R_{\mathfrak{p}}, M\right) \leq i \leq \operatorname{fd}_{R_{\mathfrak{p}}} \operatorname{Hom}_{R}\left(R_{\mathfrak{p}}, M\right)$,

where $\pi_{i}(\mathfrak{p}, M)$ is the $i$-th dual Bass number of $M$ with respect to $\mathfrak{p}$, the integer $\operatorname{cograde}_{R_{\mathfrak{p}}} \operatorname{Hom}_{R}\left(R_{\mathfrak{p}}, M\right)$ is the common length of any maximal $\operatorname{Hom}_{R}\left(R_{\mathfrak{p}}, M\right)$ quasi co-regular sequence contained in $\mathfrak{p} R_{\mathfrak{p}}$, and $\operatorname{fd}_{R_{\mathfrak{p}}} \operatorname{Hom}_{R}\left(R_{\mathfrak{p}}, M\right)$ is the flat dimension of $R_{\mathfrak{p}}$-module $\operatorname{Hom}_{R}\left(R_{\mathfrak{p}}, M\right)$ (Theorem 5.10). Besides, we also study the relations among cograde, co-dimension and flat dimension of colocalization module $\operatorname{Hom}_{R}\left(R_{\mathfrak{p}}, M\right)$.
\end{abstract}

\section{INTRODUCTION}

Let $R$ be a Noetherian ring, $M$ a $R$-module, $\mathfrak{p} \in \operatorname{Spec} R$. H. Bass 1 defined so called Bass numbers $\mu_{i}(\mathfrak{p}, M)$ by using the minimal injective resolution of $M$ for all integers $i \geq 0$, and proved that $\mu_{i}(\mathfrak{p}, M)=\operatorname{dim}_{k(\mathfrak{p})} \operatorname{Ext}_{R_{\mathfrak{p}}}^{i}\left(k(\mathfrak{p}), M_{\mathfrak{p}}\right)$. E. Enochs and J. Z. Xu defined the dual Bass numbers $\pi_{i}(\mathfrak{p}, M)$ by using the minimal flat resolution of $M$ for all $i \geq 0$ in [7, and showed that $\pi_{i}(\mathfrak{p}, M)=$ $\operatorname{dim}_{k(\mathfrak{p})} \operatorname{Tor}_{i}^{R_{\mathfrak{p}}}\left(k(\mathfrak{p}), \operatorname{Hom}_{R}\left(R_{\mathfrak{p}}, M\right)\right)$ for any cotorsion $R$-module $M$. Comparing the formulas of $\mu_{i}(\mathfrak{p}, M)$ and $\pi_{i}(\mathfrak{p}, M)$, we see that Ext is replaced by Tor and the localization $M_{\mathfrak{p}}$ is replaced by co-localization $\operatorname{Hom}_{R}\left(R_{\mathfrak{p}}, M\right)$ respectively.

The Bass numbers and dual Bass numbers are homological invariants provide a powerful means in studying structures of certain important modules and rings. The vanishing properties of Bass numbers has important roles in studying modules. It is well known that if $M$ is a finitely generated $R$-module, $\mathfrak{p} \in \operatorname{Supp}_{R} M$, then $\mu_{i}(\mathfrak{p}, M)>0$ if and only if $\operatorname{depth}_{R_{\mathfrak{p}}} M_{\mathfrak{p}} \leq i \leq \operatorname{id}_{R_{\mathfrak{p}}} M_{\mathfrak{p}}$, where $\operatorname{id}_{R_{\mathfrak{p}}} M_{\mathfrak{p}}$ is the injective dimension of $R_{\mathfrak{p}}$-module $M_{\mathfrak{p}}$. However, we known little about the vanishing properties of dual Bass numbers. In this paper, we obtain some vanishing properties of dual Bass numbers. Let $R$ be a Noetherian ring, $M$ an Artinian $R$-module, $\mathfrak{p} \in \operatorname{Cos}_{R} M$. We show that $\operatorname{cograde}_{R_{\mathfrak{p}}} \operatorname{Hom}_{R}\left(R_{\mathfrak{p}}, M\right)=\inf \left\{i \mid \pi_{i}(\mathfrak{p}, M)>0\right\}$ and

$$
\pi_{i}(\mathfrak{p}, M)>0 \Rightarrow \operatorname{cograde}_{R_{\mathfrak{p}}} \operatorname{Hom}_{R}\left(R_{\mathfrak{p}}, M\right) \leq i \leq \operatorname{fd}_{R_{\mathfrak{p}}} \operatorname{Hom}_{R}\left(R_{\mathfrak{p}}, M\right),
$$

where $\operatorname{cograde}_{R_{\mathfrak{p}}} \operatorname{Hom}_{R}\left(R_{\mathfrak{p}}, M\right)$ is the common length of any maximal $\operatorname{Hom}_{R}\left(R_{\mathfrak{p}}, M\right)$ quasi co-regular sequence contained in $\mathfrak{p} R_{\mathfrak{p}}$ and $\operatorname{fd}_{R_{\mathfrak{p}}} \operatorname{Hom}_{R}\left(R_{\mathfrak{p}}, M\right)$ is the flat dimension of $R_{\mathfrak{p}}$-module $\operatorname{Hom}_{R}\left(R_{\mathfrak{p}}, M\right)$ (Proposition 5.2, Theorem 5.10). Moreover, if $R$ is a $U$ ring (Definition 5.5) and $\operatorname{fd}_{R_{\mathfrak{p}}} \operatorname{Hom}_{R}\left(R_{\mathfrak{p}}, M\right)=t<\infty$, then $\pi_{t}(\mathfrak{p}, M)>0$.

In addition, we also study the relations among cograde, co-dimension and flat dimension of $\operatorname{Hom}_{R}\left(R_{\mathfrak{p}}, M\right)$. Let $R$ be a $U$ ring, $M$ an Artinian $R$-module. Then

$$
\operatorname{cograde}_{R_{\mathfrak{p}}} \operatorname{Hom}_{R}\left(R_{\mathfrak{p}}, M\right) \leq \operatorname{Cdim}_{R_{\mathfrak{p}}} \operatorname{Hom}_{R}\left(R_{\mathfrak{p}}, M\right) \leq \operatorname{fd}_{R_{\mathfrak{p}}} \operatorname{Hom}_{R}\left(R_{\mathfrak{p}}, M\right),
$$

where $\operatorname{Cdim}_{R_{\mathfrak{p}}} \operatorname{Hom}_{R}\left(R_{\mathfrak{p}}, M\right)$ is the co-dimension of $R_{\mathfrak{p}}$-module $\operatorname{Hom}_{R}\left(R_{\mathfrak{p}}, M\right)$. 


\section{Preliminaries}

Let $R$ be a ring, $S \subseteq R$ a multiplicative set, and $M$ an $R$-module. The $R_{S^{-}}$ module $\operatorname{Hom}_{R}\left(R_{S}, M\right)$ is called the co-localization of $M$ with respect to $S$ ([10]). The co-support of $M$ is defined by $\operatorname{Cos}_{R} M=\left\{\mathfrak{p} \in \operatorname{Spec} R \mid \operatorname{Hom}_{R}\left(R_{\mathfrak{p}}, M\right) \neq 0\right\}$. A subset $X$ of Spec $R$ is called a saturated subset of Spec $R$, if $X$ satisfies one of the following conditions: $(i) . X=\emptyset ;(i i) . X \neq \emptyset$, and $\mathrm{V}(\mathfrak{p})=\{\mathfrak{q} \in \operatorname{Spec} R \mid \mathfrak{p} \subseteq \mathfrak{q}\} \subseteq X$ for any $\mathfrak{p} \in X$. Let $\mathfrak{p}, \mathfrak{q} \in \operatorname{Spec} R$, and $\mathfrak{q} \subseteq \mathfrak{p}$. By Hom-Tensor adjunction we have

$$
\operatorname{Hom}_{R_{\mathfrak{p}}}\left(R_{\mathfrak{q}}, \operatorname{Hom}_{R}\left(R_{\mathfrak{p}}, M\right)\right) \cong \operatorname{Hom}_{R}\left(R_{\mathfrak{q}} \otimes_{R_{\mathfrak{p}}} R_{\mathfrak{p}}, M\right) \cong \operatorname{Hom}_{R}\left(R_{\mathfrak{q}}, M\right) .
$$

This implies that the co-support of any $R$-module is a saturated subset of Spec $R$.

Definition 2.1. Let $R$ be a ring, a representable $R$-module $M$ is said to be a strongly representable $R$-module, if $\left(0:_{M} I\right)=\left\{x \in M \mid I \subseteq \operatorname{Ann}_{R}(x)\right\}$ is representable for any finitely generated ideal $I$ of $R$.

It is easy to see that every Artinian module is strongly representable. However, we will show that a strongly representable module may not be an Artinian module.

Lemma 2.2. Let $R$ be a topological ring, $x_{1}, x_{2}, \cdots, x_{n} \in R$, and $M$ a linearly compact $R$-module. Then $\left(0:_{M}\left(x_{1}, x_{2}, \cdots, x_{n}\right)\right),\left(x_{1}, x_{2}, \cdots, x_{n}\right) M$, and $M /\left(x_{1}, x_{2}, \cdots, x_{n}\right) M$ are linearly compact $R$-modules.

Proof. Since $\varphi: M \stackrel{x_{1}}{\rightarrow} M$ is a continuous $R$-module homomorphism, and $\{0\}$ is a closed submodule of $M$. We have $\operatorname{ker}(\varphi)=\left(0::_{M} x_{1}\right)$ and $\operatorname{Im}(\varphi)=x_{1} M$ are both linearly compact $R$-module by [3, Lemma 2.2]. Moreover, since

$\left(0:_{M}\left(x_{1}, x_{2}, \cdots, x_{s}\right)\right)=\operatorname{ker}\left(\left(0:_{M}\left(x_{1}, x_{2}, \cdots, x_{s-1}\right)\right) \stackrel{x_{s}}{\rightarrow}\left(0:_{M}\left(x_{1}, x_{2}, \cdots, x_{s-1}\right)\right)\right)$ for $s=1,2, \cdots, n$. Then the proposition is followed by using induction on $n$.

Proposition 2.3. Let $R$ be a topological ring, $S \subseteq R$ a multiplicative set, $M$ a (resp. strongly) representable linearly compact $R$-module. Then $\operatorname{Hom}_{R}\left(R_{S}, M\right)$ is a (resp. strongly) representable $R_{S}$-module. Moreover, the functor $\operatorname{Hom}_{R}\left(R_{S},-\right)$ is an exact functor from the category of (resp. strongly) representable linearly compact $R$-modules to the category of (resp. strongly) representable $R_{S}$-modules.

Proof. Let $M$ be a representable linearly compact $R$-module. By [3, Corollary 3.3], there exists a minimal secondary representation of $M=M_{1}+\cdots+M_{n}$ and $\mathfrak{p}_{i}=$ $\sqrt{\operatorname{Ann}_{R} M_{i}}$ such that all $M_{i}$ are linearly compact. We can assume that $S \cap \mathfrak{p}_{i}=\emptyset$ $(1 \leq i \leq m)$ and $S \cap \mathfrak{p}_{i} \neq \emptyset(m+1 \leq i \leq n)$ for some integer $1 \leq m \leq n$. Then,

$$
\operatorname{Hom}_{R}\left(R_{S}, M\right)=\operatorname{Hom}_{R}\left(R_{S}, M_{1}\right)+\operatorname{Hom}_{R}\left(R_{S}, M_{2}\right)+\cdots+\operatorname{Hom}_{R}\left(R_{S}, M_{m}\right)
$$

is a minimal secondary representation of $\operatorname{Hom}_{R}\left(R_{S}, M\right)$ as a $R$-module such that $\operatorname{Hom}_{R}\left(R_{S}, M_{i}\right)$ are $\mathfrak{p}_{i}$ secondary $R$-modules by [3, Theorem 4.2]. It is easy to check that $\operatorname{Hom}_{R}\left(R_{S}, M_{i}\right)$ is a $S^{-1} \mathfrak{p}_{i}$ secondary $S^{-1} R$-module for $1 \leq i \leq m$.

If $M$ is a strongly representable linearly compact $R$-module. Let $I=\left(\frac{x_{1}}{s_{1}}, \cdots, \frac{x_{n}}{s_{n}}\right)$ be a finitely generated ideal of $R_{S}$, where $x_{i} \in R, s_{i} \in S$ for $i=1, \cdots, n$. Then

$\left(0:_{\mathrm{Hom}_{R}\left(R_{S}, M\right)} I\right)=\left(0:_{\operatorname{Hom}_{R}\left(R_{S}, M\right)}\left(\frac{x_{1}}{1}, \cdots, \frac{x_{n}}{1}\right)\right)=\operatorname{Hom}_{R}\left(R_{S}, 0:_{M}\left(x_{1}, \cdots, x_{n}\right)\right)$.

By Lemma 2.2. $\operatorname{Hom}_{R}\left(R_{S}, 0:_{M}\left(x_{1}, x_{2}, \cdots, x_{n}\right)\right)$ is a representable $R_{S}$-module.

Since co-localization preserves the exactness of short exact sequence of linearly compact modules by [3, Corollary 2.5]. Thus the functor $\operatorname{Hom}_{R}\left(R_{S},-\right)$ is an exact functor. 
Remark 2.4. Let $R$ be a ring, $M$ an Artinian $R$-module. Then $M$ is a strongly representable $R$-module (In this case, we endow $R$ and $M$ with discrete topology). Let $\mathfrak{p} \in \operatorname{Spec} R$, it is well known that $\operatorname{Hom}_{R}\left(R_{\mathfrak{p}}, M\right)$ is almost never an Artinian $R_{\mathfrak{p}}$-module. However, $\operatorname{Hom}_{R}\left(R_{\mathfrak{p}}, M\right)$ must be a strongly representable $R_{\mathfrak{p}}$-module.

Let $R$ be a ring, $M$ a $R$-module. R. N. Roberts in [11] introduced the Noetherian dimension of $M$, denoted by $\operatorname{Ndim}_{R} M$, which is defined inductively as follows: when $M=0$, put $\operatorname{Ndim}_{R} M=-1$. Then by induction, for an integer $d \geq 0$, we put $\operatorname{Ndim}_{R} M=d$, if $\operatorname{Ndim}_{R} M=d$ is false and for every ascending chain $M_{0} \subseteq M_{1} \subseteq \cdots$ of submodules of $M$, there exists a positive integer $n_{0}$ such that $\operatorname{Ndim}_{R}\left(M_{n+1} / M_{n}\right)<d$ for all $n>n_{0}$. Therefore $\operatorname{Ndim}_{R} M=0$ if and only if $M$ is a non-zero Noetherian $R$-module. On the other hand, for any representable linearly compact module $M$ over some Noetherian topological ring $R, \operatorname{Att}_{R} M, \operatorname{Cos}_{R} M$, $\mathrm{Ann}_{R} M$ have same minimal elements by [3, Corollary 4.3]. Thus

$$
\sup \left\{\operatorname{dim} R / \mathfrak{p} \mid \mathfrak{p} \in \operatorname{Cos}_{R} M\right\}=\sup \left\{\operatorname{dim} R / \mathfrak{p} \mid \mathfrak{p} \in \operatorname{Att}_{R} M\right\}=\operatorname{dim} R / \operatorname{Ann}_{R} M .
$$

Hence, we introduce the following definition.

Definition 2.5. Let $R$ be a ring, and $M$ an $R$-module. The co-dimension of $M$ is defined as the integer (possibly infinite)

$$
\operatorname{Cdim}_{R} M=\sup \left\{\operatorname{dim} R / \mathfrak{p} \mid \mathfrak{p} \in \operatorname{Cos}_{R} M\right\} .
$$

We except that $\operatorname{Ndim}_{R} M=\operatorname{Cdim}_{R} M$ for any Artinian $R$-modules $M$. Unfortunately, this equality does not holds in general. N. T. Cuong and L. T. Nhan [4, Example 4.1] showed that exists an Artinian module $M$ over a Noetherian local ring $(R, \mathfrak{m})$ such that $\operatorname{Ndim}_{R} M<\operatorname{Cdim}_{R} M$. However, we have the following result.

Proposition 2.6. Let $R$ be a Noetherian ring, $M$ an Artinian $R$-module such that $\operatorname{Ann}_{R}(0: M \mathfrak{p})=\mathfrak{p}$ for any $\mathfrak{p} \in \mathrm{V}\left(\operatorname{Ann}_{R} M\right)$. Then $\operatorname{Ndim}_{R} M=\operatorname{Cdim}_{R} M$.

Proof. Since $\operatorname{Cdim}_{R} M=\operatorname{dim} R / \operatorname{Ann}_{R} M$ for any Artinian $R$-module $M$. It is enough to show that $\operatorname{Ndim}_{R} M=\operatorname{dim} R / \operatorname{Ann}_{R} M$.

By [4, Proposition 2.4], we have the inequality $\operatorname{Ndim}_{R} M \leq \operatorname{dim} R / \operatorname{Ann}_{R} M$. On the other hand, for any ideal $\mathfrak{a} \subsetneq R$, we have $\left(\mathfrak{a}+\operatorname{Ann}_{R} M\right) \subseteq \operatorname{Ann}_{R}\left(0:_{M} \mathfrak{a}\right)$, and $\operatorname{Ann}_{R}\left(0:_{M} \mathfrak{a}\right) \subseteq \operatorname{Ann}_{R}\left(0:_{M} \mathfrak{p}\right)=\mathfrak{p}$ for any $\left(\mathfrak{a}+\operatorname{Ann}_{R} M\right) \subseteq \mathfrak{p} \in$ Spec $R$ by hypothesis. Thus $\sqrt{\mathfrak{a}+\operatorname{Ann}_{R} M}=\sqrt{\operatorname{Ann}_{R}\left(0:_{M} \mathfrak{a}\right)}$. Let $\operatorname{Ndim}_{R} M=d$. Then there exist elements $x_{1}, \cdots, x_{d} \in J(M)=\bigcap_{\mathfrak{m}_{\in} \operatorname{Supp}_{R} M} \mathfrak{m}$ such that $\left(0:_{M}\left(x_{1}, \cdots, x_{d}\right)\right) \neq 0$ have finite length by [13, Theorem 4.1]. Let $\mathfrak{a}=\left(x_{1}, \cdots, x_{d}\right)$. Then $0=\operatorname{dim}_{R}\left(0:_{M}\left(x_{1}, \cdots, x_{d}\right)\right)=\operatorname{dim}_{R} R /\left(\left(x_{1}, \cdots, x_{d}\right)+\operatorname{Ann}_{R} M\right) \geq \operatorname{dim} R / \operatorname{Ann}_{R} M-d$. Hence, $\operatorname{Cdim}_{R} M=\operatorname{Ndim}_{R} M$.

Remark 2.7. Let $R$ be a topological ring (not necessarily Noetherian), $S \subseteq R$ a multiplicative set, $M$ a representable linearly compact $R$-module. Then by the proof of Proposition 2.3, we have

$$
\begin{aligned}
\operatorname{Cdim}_{S^{-1}} \operatorname{Hom}_{R}\left(S^{-1} R, M\right) & =\sup \left\{\operatorname{dim} S^{-1} R / S^{-1} \mathfrak{p} \mid \mathfrak{p} \in \operatorname{Cos}_{R} M, \mathfrak{p} \cap S=\emptyset\right\} \\
& =\sup \left\{\operatorname{dim} S^{-1} R / S^{-1} \mathfrak{p} \mid \mathfrak{p} \in \operatorname{Att}_{R} M, \mathfrak{p} \cap S=\emptyset\right\} .
\end{aligned}
$$

Let $\mathfrak{p} \in \operatorname{Cos}_{R} M$, we denote

$$
\operatorname{ht}_{M} \mathfrak{p}=\sup \left\{n \mid \mathfrak{p}_{0} \subsetneq \mathfrak{p}_{1} \subsetneq \cdots \subsetneq \mathfrak{p}_{n}=\mathfrak{p}, \mathfrak{p}_{i} \in \operatorname{Cos}_{R} M \text { for } i=0,1, \cdots, n\right\} .
$$

It is obvious that $\operatorname{ht}_{M} \mathfrak{p}=\operatorname{Cdim}_{R_{\mathfrak{p}}} \operatorname{Hom}_{R}\left(R_{\mathfrak{p}}, M\right)$. 


\section{Filter CO-Regular Sequence And COGRade}

Definition 3.1. Let $R$ be a ring, $X \subseteq$ Spec $R$ and $M$ an $R$-module. A sequence $x_{1}, x_{2}, \cdots, x_{n} \in R$ is called an $M$-filter co-regular sequence with respect to $X$, if $\operatorname{Cos}_{R}\left(\left(0:_{M}\left(x_{1}, \cdots, x_{i-1}\right)\right) / x_{i}\left(0:_{M}\left(x_{1}, \cdots, x_{i-1}\right)\right)\right) \subseteq X$ for $i=1,2, \cdots, n$.

For any $R$-module $M$, any $M$-quasi co-regular sequence is a $M$-filter co-regular sequence with respect to $\emptyset$. Moreover, if $M$ is a linearly compact $R$-module, then the converse is also true by [2, Corollary 4.3].

Proposition 3.2. Let $R$ be a Noetherian topological ring, $X$ a saturated set of Spec $R$ and $M$ a representable linearly compact $R$-module. Then $x \in R$ is a $M$ filter co-regular element with respect to $X$ if and only if $x \in R-\bigcup_{\mathfrak{p} \in \operatorname{Att}_{R} M-X} \mathfrak{p}$.

Proof. Let $x \in R$ be a $M$-filter co-regular element with respect to $X$. Suppose that there exists a prime ideal $\mathfrak{p} \in \operatorname{Att}_{R} M-X$ such that $x \in \mathfrak{p}$. Then we have $\mathfrak{p} \in \operatorname{Att}_{R} M \cap \mathrm{V}((x))=\operatorname{Att}_{R}(M / x M)$ by [3, Theorem 4.5]. This contradicts to $\operatorname{Att}_{R}(M / x M) \subseteq \operatorname{Cos}_{R}(M / x M) \subseteq X$. Hence, $x \in R-\bigcup_{\mathfrak{p} \in \operatorname{Att}_{R} M-X} \mathfrak{p}$.

Let $M=M_{1}+M_{2}+\cdots+M_{n}$ be a minimal secondary representation of $M$, where $M_{i}$ is a $\mathfrak{p}_{i}$ secondary $R$-module for $i=1,2, \cdots, n$, and $\operatorname{Att}_{R} M=$ $\left\{\mathfrak{p}_{1}, \mathfrak{p}_{2}, \cdots, \mathfrak{p}_{n}\right\}$. Let $x \in R-\bigcup_{\mathfrak{p} \in \operatorname{Att}_{R} M-X} \mathfrak{p}$. If $x \in R-\bigcup_{\mathfrak{p} \in \operatorname{Att}_{R} M} \mathfrak{p}$, then $M=x M$.

Thus $\operatorname{Cos}_{R}(M / x M)=\emptyset \subseteq X$. Hence, $x \in R$ is a $M$-filter co-regular element with respect to $X$. If there is some $1 \leq m \leq n$, such that $x \in \mathfrak{p}_{i} \in \operatorname{Att}_{R} M \cap X$ for $1 \leq i \leq m$ and $x \in R-\mathfrak{p}_{i}$ for $m+1 \leq i \leq n$, then

$x \bar{M}=x\left(M_{1}+M_{2}+\cdots+M_{n}\right)=x M_{1}+x M_{2}+\cdots+x M_{m}+M_{m+1} \cdots+M_{n}$, and $M / x M=\left(M_{1}+M_{2}+\cdots+M_{n}\right) /\left(x M_{1}+x M_{2}+\cdots+x M_{m}+M_{m+1} \cdots+M_{n}\right)$ $\cong\left(M_{1}+M_{2}+\cdots+M_{m}\right) /\left(\left(M_{1}+\cdots+M_{m}\right) \cap\left(x M_{1}+\cdots+x M_{m}+M_{m+1} \cdots+M_{n}\right)\right)$. Thus $\operatorname{Att}_{R}(M / x M) \subseteq \operatorname{Att}_{R}\left(M_{1}+\cdots+M_{m}\right)=\left\{\mathfrak{p}_{1}, \cdots, \mathfrak{p}_{m}\right\} . \operatorname{Thus}_{\operatorname{Cos}_{R}}(M / x M) \subseteq$ $\bigcup_{1 \leq i \leq m} \mathrm{~V}\left(\mathfrak{p}_{i}\right) \subseteq X$

Corollary 3.3. Let $R$ be a Noetherian topological ring, and $X$ a saturated subset of Spec $R$. Let $I$ be an ideal of $R$ and $M$ a representable linearly compact $R$-module. Then $\operatorname{Cos}_{R}(M / I M) \subseteq X$ if and only if there is a $M$-filter co-regular element with respect to $X$ contained in $I$.

Proof. Since $M / I M$ is a representable linearly compact $R$-module, then by 3 , Theorem 4.5] and Proposition 3.2, we have

$$
\begin{aligned}
\operatorname{Cos}_{R}(M / I M) \subseteq X & \Leftrightarrow \operatorname{Att}_{R}(M / I M) \subseteq X \\
& \Leftrightarrow \operatorname{Att}_{R} M \cap \mathrm{V}(I) \subseteq X \\
& \Leftrightarrow I \nsubseteq \bigcup_{\mathfrak{p} \in \operatorname{Att}_{R} M-X} \mathfrak{p} \\
& \Leftrightarrow I \text { contains a } M \text {-filter co-regular element with respect to } X .
\end{aligned}
$$

Proposition 3.4. Let $R$ be a topological ring, $X \subseteq$ Spec $R$ and $M$ a linearly compact $R$-module. Then $x_{1}, x_{2}, \cdots, x_{n} \in R$ is a $M$-filter co-regular sequence with respect to $X$ if and only if $\frac{x_{1}}{1}, \cdots, \frac{x_{n}}{1} \in R_{\mathfrak{p}}$ is a $\operatorname{Hom}_{R}\left(R_{\mathfrak{p}}, M\right)$-quasi co-regular sequence for any $\mathfrak{p} \in \operatorname{Cos}_{R} M-X$. 
Proof. For any $1 \leq i \leq n$, consider the exact sequence of $R$-modules

$$
\left(0:_{M}\left(x_{1}, \cdots, x_{i-1}\right)\right) \stackrel{x_{i}}{\rightarrow}\left(0:_{M}\left(x_{1}, \cdots, x_{i-1}\right)\right) \rightarrow \operatorname{coker}\left(x_{i}\right) \rightarrow 0 .
$$

By [3, Corollary 2.5], we have an exact sequence of $R_{\mathfrak{p}}$-modules

$$
\begin{gathered}
\operatorname{Hom}_{R}\left(R_{\mathfrak{p}}, 0:_{M}\left(x_{1}, \cdots, x_{i-1}\right)\right) \stackrel{\frac{x_{i}}{\rightarrow}}{\rightarrow} \operatorname{Hom}_{R}\left(R_{\mathfrak{p}}, 0:_{M}\left(x_{1}, \cdots, x_{i-1}\right)\right) \rightarrow \\
\operatorname{Hom}_{R}\left(R_{\mathfrak{p}}, \operatorname{coker}\left(x_{i}\right)\right) \rightarrow 0,
\end{gathered}
$$

for any $\mathfrak{p} \in \operatorname{Spec} R-X$.

Since $\operatorname{Hom}_{R}\left(R_{\mathfrak{p}}, 0:_{M}\left(x_{1}, \cdots, x_{i-1}\right)\right)=\left(0:_{\operatorname{Hom}_{R}\left(R_{\mathfrak{p}}, M\right)}\left(\frac{x_{1}}{1}, \cdots, \frac{x_{i-1}}{1}\right)\right)$, we have the following equivalent statements:

The sequence $x_{1}, x_{2}, \cdots, x_{n}$ is an M-filter co-regular sequence with respect to $X$. $\Leftrightarrow\left(0:_{\operatorname{Hom}_{R}\left(R_{\mathfrak{p}}, M\right)}\left(\frac{x_{1}}{1}, \cdots, \frac{x_{i-1}}{1}\right)\right) \stackrel{\frac{x_{i}}{\rightarrow}}{\rightarrow}\left(0:_{\operatorname{Hom}_{R}\left(R_{\mathfrak{p}}, M\right)}\left(\frac{x_{1}}{1}, \cdots, \frac{x_{i-1}}{1}\right)\right)$ is surjective for any $\mathfrak{p} \in \operatorname{Cos}_{R} M-X, i=1,2, \cdots, n$. $\Leftrightarrow \frac{x_{1}}{1}, \cdots, \frac{x_{n}}{1} \in R_{\mathfrak{p}}$ is a $\operatorname{Hom}_{R}\left(R_{\mathfrak{p}}, M\right)$ quasi co-regular sequence for any $\mathfrak{p} \in \operatorname{Cos}_{R} M-X$.

Definition 3.5. Let $R$ be a ring, $X \subseteq$ Spec $R$. A $R$-module sequence

$$
M_{0} \stackrel{f_{1}}{\rightarrow} M_{1} \stackrel{f_{2}}{\longrightarrow} \ldots \stackrel{f_{n-1}}{\rightarrow} M_{n-1} \stackrel{f_{n}}{\rightarrow} M_{n}
$$

is called a quasi exact sequence of $R$-modules with respect to $X$, if $f_{i+1} \circ f_{i}=0$, and $\operatorname{Cos}_{R}\left(\operatorname{ker}\left(f_{i+1}\right) / \operatorname{Im}\left(f_{i}\right)\right) \subseteq X$ for $i=1,2, \cdots, n-1$.

Lemma 3.6. Let $R$ be a topological ring, $X \subseteq$ Spec $R$ and a complex of $R$-modules

$$
C_{\bullet}: \cdots \stackrel{f_{i-2}}{\rightarrow} M_{i-1} \stackrel{f_{i-1}}{\rightarrow} M_{i} \stackrel{f_{i}}{\rightarrow} M_{i+1} \stackrel{f_{i+1}}{\rightarrow} \cdots
$$

where $M_{i}$ are linearly compact $R$-module, $f_{i}$ are continuous homomorphisms for all integers $i \in \mathbb{Z}$. Then $C_{\bullet}$ is a quasi exact sequence of $R$-modules with respect to $X$ if and only if $\operatorname{Hom}_{R}\left(R_{\mathfrak{p}}, C_{\bullet}\right)$ is an exact sequence of $R_{\mathfrak{p}}$-modules for any $\mathfrak{p} \in \operatorname{Spec} R-X$.

Proof. Since the kernel and cokernel of a continuous homomorphism between linearly compact $R$-modules are linearly compact $R$-modules, and the co-localization preserves the exactness of linearly compact $R$-modules ([3, Corollary 2.5]), we get

$$
\begin{aligned}
\mathrm{H}_{i}\left(\operatorname{Hom}_{R}\left(R_{\mathfrak{p}}, C_{\bullet}\right)\right) & =\operatorname{ker}\left(\operatorname{Hom}_{R}\left(R_{\mathfrak{p}}, d_{i}\right)\right) / \operatorname{Im}\left(\operatorname{Hom}_{R}\left(R_{\mathfrak{p}}, d_{i-1}\right)\right) \\
& \cong \operatorname{Hom}_{R}\left(R_{\mathfrak{p}}, \operatorname{ker}\left(d_{i}\right)\right) / \operatorname{Hom}_{R}\left(R_{\mathfrak{p}}, \operatorname{Im}\left(d_{i-1}\right)\right) \\
& \cong \operatorname{Hom}_{R}\left(R_{\mathfrak{p}}, \mathrm{H}_{i}\left(C_{\bullet}\right)\right), \text { for all } i \in \mathbb{Z} \text { and for any } \mathfrak{p} \in \operatorname{Spec} R .
\end{aligned}
$$

Thus, $C$. is a quasi exact sequence of $R$-modules with respect to $X$ if and only if $\operatorname{Hom}_{R}\left(R_{\mathfrak{p}}, \mathrm{H}_{i}\left(C_{\bullet}\right)\right)=0$, for all $i \in \mathbb{Z}, \mathfrak{p} \in \operatorname{Spec} R-X$. Equivalently, $\operatorname{Hom}_{R}\left(R_{\mathfrak{p}}, C_{\bullet}\right)$ is an exact sequence of $R_{\mathfrak{p}}$-modules for all $\mathfrak{p} \in \operatorname{Spec} R-X$.

The following theorem is the main result of this section.

Theorem 3.7. Let $R$ be a Noetherian topological ring, $X$ a saturated subset of Spec $R$. Let $I$ be an ideal of $R$ and $M$ a strongly representable linearly compact $R$-module. Then for a given integer $n>0$ the following conditions are equivalent:

(i). $\operatorname{Cos}_{R}\left(\operatorname{Tor}_{i}^{R}(N, M)\right) \subseteq X$ for all $0 \leq i<n$ and for any finitely generated $R$-module $N$ with $\operatorname{Supp}_{R} N \subseteq \mathrm{V}(I)$;

(ii). $\operatorname{Cos}_{R}\left(\operatorname{Tor}_{i}^{R}(R / I, M)\right) \subseteq X$ for all $0 \leq i<n$;

(iii). There exists a $M$-filter co-regular sequence with respect to $X$ of length $n$ contained in $I$. 
Proof. $(i) \Rightarrow($ ii $)$. Obviously.

(ii $) \Rightarrow($ iii $)$. We use induction on $n$. For the case $n=1$ we have $R / I \otimes_{R} M=$ $M / I M$ and $\operatorname{Cos}_{R}(M / I M) \subseteq X$. Then the assertion is followed by Corollary 3.3 Suppose that $n>1$, and the statement holds up to $n-1$. Let $x_{1} \in I$ be a $M$-filter co-regular element with respect to $X$, and $M_{1}=\left(0:_{M} x_{1}\right)$. Then $M_{1}$ is a strongly representable linearly compact $R$-module. Moreover, we get the following quasi exact sequence of $R$-modules with respect to $X, 0 \rightarrow M_{1} \rightarrow M \stackrel{x_{1}}{\rightarrow} M \rightarrow 0$. For $\mathfrak{p} \in$ Spec $R-X$, by Lemma 3.6 we have an exact sequence of $R_{\mathfrak{p}}$-modules

$$
0 \rightarrow \operatorname{Hom}_{R}\left(R_{\mathfrak{p}}, M_{1}\right) \rightarrow \operatorname{Hom}_{R}\left(R_{\mathfrak{p}}, M\right) \stackrel{\frac{x_{1}}{\rightarrow}}{\rightarrow} \operatorname{Hom}_{R}\left(R_{\mathfrak{p}}, M\right) \rightarrow 0 .
$$

From this we can get a long exact sequence of $R_{\mathfrak{p}}$-modules

$$
\begin{gathered}
\cdots \rightarrow \operatorname{Tor}_{1}^{R_{\mathfrak{p}}}\left(R_{\mathfrak{p}} / I R_{\mathfrak{p}}, \operatorname{Hom}_{R}\left(R_{\mathfrak{p}}, M\right)\right) \rightarrow R_{\mathfrak{p}} / I R_{\mathfrak{p}} \otimes_{R_{\mathfrak{p}}} \operatorname{Hom}_{R}\left(R_{\mathfrak{p}}, M_{1}\right) \rightarrow \\
R_{\mathfrak{p}} / I R_{\mathfrak{p}} \otimes_{R_{\mathfrak{p}}} \operatorname{Hom}_{R}\left(R_{\mathfrak{p}}, M\right) \stackrel{\stackrel{x_{1}}{\rightarrow}}{\rightarrow} R_{p} / I R_{\mathfrak{p}} \otimes_{R_{\mathfrak{p}}} \operatorname{Hom}_{R}\left(R_{\mathfrak{p}}, M\right) \rightarrow 0 .
\end{gathered}
$$

Since $\operatorname{Hom}_{R}\left(R_{\mathfrak{p}}, \operatorname{Tor}_{i}^{R}(R / I, M)\right) \cong \operatorname{Tor}_{i}^{R_{\mathfrak{p}}}\left(R_{\mathfrak{p}} / I R_{\mathfrak{p}}, \operatorname{Hom}_{R}\left(R_{\mathfrak{p}}, M\right)\right)$ by the proof of [2, Theorem 3.6], we get $\operatorname{Cos}_{R}\left(\operatorname{Tor}_{i}^{R}\left(R / I, M_{1}\right)\right) \subseteq X$ for all $0 \leq i<n-1$. Then the statement is followed by the induction hypothesis.

(iii) $\Rightarrow(i)$. Let $x_{1}, x_{2}, \cdots, x_{n} \in I$ be a $M$-filter co-regular sequence with respect to $X$ of length $n$. Since $\mathrm{V}\left(\operatorname{Ann}_{R} N\right)=\operatorname{Supp}_{R} N \subseteq \mathrm{V}(I)$, there exists $m>0$ such that $x_{1}^{m} \in \operatorname{Ann}_{R} N$. We use induction on $n$.

For the case $n=1$, we take co-localization to the homomorphism of $R$-modules $M \stackrel{x_{7}}{\rightarrow} M$, we get an exact sequence of $R_{\mathfrak{p}}$-modules

$$
\operatorname{Hom}_{R}\left(R_{\mathfrak{p}}, M\right) \stackrel{\frac{x_{1}}{\rightarrow}}{\rightarrow} \operatorname{Hom}_{R}\left(R_{\mathfrak{p}}, M\right) \rightarrow 0
$$

for any $\mathfrak{p} \in$ Spec $R-X$. It follows that we have an exact sequence of $R_{\mathfrak{p}}$-modules

$$
N_{\mathfrak{p}} \otimes_{R_{\mathfrak{p}}} \operatorname{Hom}_{R}\left(R_{\mathfrak{p}}, M\right) \stackrel{\frac{x_{1}}{\rightarrow}}{\rightarrow} N_{\mathfrak{p}} \otimes_{R_{\mathfrak{p}}} \operatorname{Hom}_{R}\left(R_{\mathfrak{p}}, M\right) \rightarrow 0 .
$$

Since $x_{1}^{m} \in \operatorname{Ann}_{R}(N)$, we have $\left(\frac{x_{1}}{1}\right)^{m} \in \operatorname{Ann}_{R_{\mathfrak{p}}}\left(N_{\mathfrak{p}}\right)$. Then

$$
\operatorname{Hom}_{R}\left(R_{\mathfrak{p}}, N \otimes_{R} M\right) \cong N_{p} \otimes_{R_{\mathfrak{p}}} \operatorname{Hom}_{R}\left(R_{\mathfrak{p}}, M\right)=0
$$

for all $\mathfrak{p} \in$ Spec $R-X$. Hence $\operatorname{Cos}_{R}\left(N \otimes_{R} M\right) \subseteq X$.

Suppose that $n>1$, and the statement holds up to $n-1$. Set $M_{1}=\left(0:_{M} x_{1}\right)$. Consider the quasi exact sequence of $R$-modules with respect to $X$

$$
0 \rightarrow M_{1} \rightarrow M \stackrel{x_{7}}{\rightarrow} M \rightarrow 0 .
$$

Then for any $\mathfrak{p} \in$ Spec $R-X$, by Lemma 3.6. we have an exact sequence

$$
0 \rightarrow \operatorname{Hom}_{R}\left(R_{\mathfrak{p}}, M_{1}\right) \rightarrow \operatorname{Hom}_{R}\left(R_{\mathfrak{p}}, M\right) \stackrel{\frac{x_{1}}{1}}{\rightarrow} \operatorname{Hom}_{R}\left(R_{\mathfrak{p}}, M\right) \rightarrow 0 .
$$

Therefore, for any integer $i \geq 0$, we have an exact sequence of $R_{\mathfrak{p}}$-modules

$$
\begin{gathered}
\operatorname{Tor}_{i+1}^{R_{\mathfrak{p}}}\left(N_{\mathfrak{p}}, \operatorname{Hom}_{R}\left(R_{\mathfrak{p}}, M\right)\right) \stackrel{\frac{x_{1}}{\rightarrow}}{\rightarrow} \operatorname{Tor}_{i+1}^{R_{\mathfrak{p}}}\left(N_{\mathfrak{p}}, \operatorname{Hom}_{R}\left(R_{\mathfrak{p}}, M\right)\right) \\
\rightarrow \operatorname{Tor}_{i}^{R_{\mathfrak{p}}}\left(N_{\mathfrak{p}}, \operatorname{Hom}_{R}\left(R_{\mathfrak{p}}, M_{1}\right)\right) .
\end{gathered}
$$

By induction hypothesis we have $\operatorname{Tor}_{i}^{R_{\mathfrak{p}}}\left(N_{\mathfrak{p}}, \operatorname{Hom}_{R}\left(R_{\mathfrak{p}}, M_{1}\right)\right)=0$ for any $\mathfrak{p} \in$ Spec $R-X$ and for any $0 \leq i<n-1$. Moreover, $\left(\frac{x_{1}}{1}\right)^{m} \in \operatorname{Ann}_{R_{\mathfrak{p}}}\left(N_{\mathfrak{p}}\right)$, since $x_{1}^{m} \in \operatorname{Ann}_{R}(N)$. Then $\operatorname{Tor}_{i+1}^{R_{\mathfrak{p}}}\left(N_{\mathfrak{p}}, \operatorname{Hom}_{R}\left(R_{\mathfrak{p}}, M\right)\right)=0$ for any $\mathfrak{p} \in$ Spec $R-X$ and any $0 \leq i<n-1$. Hence, $\operatorname{Cos}_{R}\left(\operatorname{Tor}_{i}^{R}(N, M)\right) \subseteq X$ for any $0 \leq i<n$. 
Definition 3.8. Let $R$ be a Noetherian topological ring, $X$ a saturated subset of Spec $R, I$ an ideal of $R$ and $M$ a strongly representable linearly compact $R$ module. Then we define $M$-filter cograde with respect to $X$ contained in $I$ as the integer (possibly infinite)

$$
\operatorname{cograde}_{X}(I, M)=\inf \left\{i \mid \operatorname{Cos}_{R}\left(\operatorname{Tor}_{i}^{R}(R / I, M)\right) \nsubseteq X X\right\} .
$$

By Theorem 3.7, it is easy to obtain the following facts:

(1). Any $M$-filter co-regular sequence with respect to $X$ contained in $I$ of finite length can be extended to a maximal one.

(2). $\operatorname{cograde}_{X}(I, M)$ is possibly equal to $\infty$. e.g. if $X=\operatorname{Cos}_{R} M \cap \mathrm{V}(I)$.

(3). $\operatorname{cograde}_{X}(I, M)$ is the common length of each maximal $M$-filter co-regular sequence with respect to $X$ contained in $I$.

(4). Let $I^{\prime}$ be another ideal of $R$ such that $\sqrt{I}=\sqrt{I^{\prime}}$, then $\operatorname{cograde}_{X}(I, M)=$ $\operatorname{cograde}_{X}\left(I^{\prime}, M\right)$.

(5). Let $R$ be a Noetherian topological ring, $M$ a strongly representable linearly compact $R$-module. Then $\operatorname{cograde}_{\emptyset}(I, M)=\inf \left\{i \mid \operatorname{Tor}_{i}^{R}(R / I, M) \neq 0\right\}$ equals to the length of any maximal $M$ co-regular sequence in $I$.

(6). Let $(R, \mathfrak{m})$ be a Noetherian local ring, $I \subseteq R$ an ideal and $M$ an Artinian $R$-module. Then for any $i \geq 0$, $\operatorname{Tor}_{i}^{R}(R / I, M)$ is an Artinian $R$ module. Since $\operatorname{Cos}_{R} M \subseteq\{\mathfrak{m}\}$ iff $M$ is a finite length $R$-module, we have $\operatorname{cograde}_{\{\mathfrak{m}\}}(I, M)=\inf \left\{i \mid \operatorname{Tor}_{i}^{R}(R / I, M)\right.$ is not a finite length $R-$ module\}.

\section{Filter co-Regular Sequence and Quasi Co-Regular sequence}

Now, we will generalize some results of [12] and [13], and extend the theory of quasi co-regular sequence to some class of modules which are not necessarily Artinian.

Proposition 4.1. Let $R$ be a Noetherian topological ring, $M$ a strongly representable linearly compact $R$-module, $\mathfrak{p} \in$ Spec $R$, and $I$ an ideal of $R_{\mathfrak{p}}$. Then for a given integer $n>0$ the following statements are equivalent:

(1). $\operatorname{Tor}_{i}^{R_{\mathfrak{p}}}\left(N, \operatorname{Hom}_{R}\left(R_{\mathfrak{p}}, M\right)\right)=0$ for all $0 \leq i<n$ and for any finitely generated $R_{\mathfrak{p}}$-module $N$ with $\operatorname{Supp}_{R_{\mathfrak{p}}} N \subseteq \mathrm{V}(I)$.

(2). $\operatorname{Tor}_{i}^{R_{\mathfrak{p}}}\left(R_{\mathfrak{p}} / I, \operatorname{Hom}_{R}\left(R_{\mathfrak{p}}, M\right)\right)=0$ for any $0 \leq i<n$.

(3). There exists a $\operatorname{Hom}_{R}\left(R_{\mathfrak{p}}, M\right)$-quasi co-regular sequence of length $n$ contained in $I$.

Proof. If $\mathfrak{p} \notin \operatorname{Cos}_{R} M$, this Proposition is obvious. Now we assume that $\mathfrak{p} \in \operatorname{Cos}_{R} M$.

$(1) \Rightarrow(2)$. Obviously.

$(2) \Rightarrow(3)$. We use induction on $n$. For the case $n=1$, let $J \subseteq R$ be an ideal such that $I=J R_{\mathfrak{p}}$. Since $\left(R_{\mathfrak{p}} / I\right) \otimes_{R_{\mathfrak{p}}} \operatorname{Hom}_{R}\left(R_{\mathfrak{p}}, M\right)=\operatorname{Hom}_{R}\left(R_{\mathfrak{p}},(R / J) \otimes_{R} M\right)=0$, we have $\mathfrak{p} \notin \operatorname{Cos}_{R}(M / J M)$. Let $X=\{\mathfrak{q} \mid \mathfrak{q} \in \operatorname{Spec} R, \mathfrak{q} \nsubseteq \mathbb{p}\}$. Then $X$ is a saturated subset of Spec $R$. Obviously, $\operatorname{Cos}_{R}(M / J M) \subseteq X$ and there exists a $M$-filter co-regular element $x \in J$ with respect to $X$ by Corollary 3.3 . Then by Proposition 3.4 we know that $\frac{x}{1} \in I$ is a $\operatorname{Hom}_{R}\left(R_{\mathfrak{p}}, M\right)$-quasi co-regular element.

The proof of the case $n>1$ and the proof of $(3) \Rightarrow(1)$ is similar to the proof of Theorem 3.7. We will not go into details. 
With the same notation of Proposition 4.1, we denote

$$
\operatorname{cograde}_{R_{\mathfrak{p}}}\left(I, \operatorname{Hom}_{R}\left(R_{\mathfrak{p}}, M\right)\right)=\inf \left\{i \mid \operatorname{Tor}_{i}^{R_{\mathfrak{p}}}\left(R_{\mathfrak{p}} / I, \operatorname{Hom}_{R}\left(R_{\mathfrak{p}}, M\right)\right) \neq 0\right\} .
$$

In particular, if $I=\mathfrak{p} R_{\mathfrak{p}}$, we simply write $\operatorname{cograde}_{R_{\mathfrak{p}}} \operatorname{Hom}_{R}\left(R_{\mathfrak{p}}, M\right)$. By Proposition 4.1, we notice that $\operatorname{cograde}_{R_{\mathfrak{p}}}\left(I, \operatorname{Hom}_{R}\left(R_{\mathfrak{p}}, M\right)\right)$ equals to the length of any maximal $\operatorname{Hom}_{R}\left(R_{\mathfrak{p}}, M\right)$-quasi co-regular sequence contained in $I$, and any $\operatorname{Hom}_{R}\left(R_{\mathfrak{p}}, M\right)$ quasi co-regular sequence contained in $I$ can be extended to a maximal one.

Proposition 4.2. Let $R$ be a Noetherian topological ring, $X$ a saturated subset of Spec $R$, and $I \subsetneq R$ a proper ideal of $R$, and $M$ a strongly representable linearly compact $R$-module. Then

$$
\begin{aligned}
\operatorname{cograde}_{X}(I, M) & =\inf \left\{\operatorname{cograde}_{R_{\mathfrak{p}}} \operatorname{Hom}_{R}\left(R_{\mathfrak{p}}, M\right) \mid \mathfrak{p} \in \operatorname{Cos}_{R} M \cap \mathrm{V}(I)-X\right\} \\
= & \inf \left\{\operatorname{cograde}_{R_{\mathfrak{p}}}\left(I R_{\mathfrak{p}}, \operatorname{Hom}_{R}\left(R_{\mathfrak{p}}, M\right)\right) \mid \mathfrak{p} \in \operatorname{Cos}_{R} M \cap \mathrm{V}(I)-X\right\} .
\end{aligned}
$$

Proof. If $\operatorname{cograde}_{X}(I, M)=\infty$, there is nothing to prove. So we assume that $\operatorname{cograde}_{X}(I, M)<\infty$. If $\operatorname{Cos}_{R} M \cap \mathrm{V}(I) \subseteq X$, then $\operatorname{Cos}_{R}\left(\operatorname{Tor}_{i}^{R}(R / I, M)\right) \subseteq X$ for

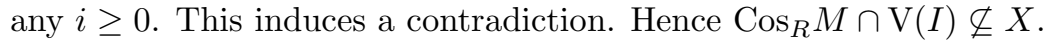

(i). Let $\mathfrak{p} \in \operatorname{Cos}_{R} M \cap \mathrm{V}(I)-X$. Then we have

$$
\operatorname{cograde}_{X}(I, M) \leq \operatorname{cograde}_{X}(\mathfrak{p}, M) \leq \operatorname{cograde}_{R_{\mathfrak{p}}} \operatorname{Hom}_{R}\left(R_{\mathfrak{p}}, M\right) .
$$

Let $x_{1}, x_{2}, \cdots, x_{n}$ be a maximal $M$-filter co-regular sequence with respect to $X$ contained in $I$. So there exists no $\left(0:_{M}\left(x_{1}, x_{2}, \cdots, x_{n}\right)\right)$-filter co-regular element contained in $I$. By Proposition 3.2, there exists $\mathfrak{p} \in \operatorname{Att}_{R}\left(0:_{M}\left(x_{1}, x_{2}, \cdots, x_{n}\right)\right)-X$ such that $I \subseteq \mathfrak{p}$. Then $\mathfrak{p} \in \operatorname{Cos}_{R} M \cap \mathrm{V}(I)-X$, and

$$
\mathfrak{p} R_{\mathfrak{p}} \in \operatorname{Att}_{R_{\mathfrak{p}}} \operatorname{Hom}_{R}\left(R_{\mathfrak{p}}, 0:_{M}\left(x_{1}, x_{2}, \cdots, x_{n}\right)\right) .
$$

Since $\operatorname{Hom}_{R}\left(R_{\mathfrak{p}}, 0:_{M}\left(x_{1}, x_{2}, \cdots, x_{n}\right)\right) \cong\left(0: \operatorname{Hom}_{R}\left(R_{\mathfrak{p}}, M\right)\left(\frac{x_{1}}{1}, \cdots, \frac{x_{n}}{1}\right)\right)$. Thus $\frac{x_{1}}{1}, \cdots, \frac{x_{n}}{1} \in R_{\mathfrak{p}}$ is a maximal $\operatorname{Hom}_{R}\left(R_{\mathfrak{p}}, M\right)$ co-regular sequence. Hence,

$$
\operatorname{cograde}_{X}(I, M)=\operatorname{Min}\left\{\operatorname{cograde}_{R_{\mathfrak{p}}} \operatorname{Hom}_{R}\left(R_{\mathfrak{p}}, M\right) \mid \mathfrak{p} \in \operatorname{Cos}_{R} M \cap \mathrm{V}(I)-X\right\} .
$$

(ii). Assume that $\operatorname{cograde}_{X}(I, M)=n$. Then $\operatorname{Tor}_{i}^{R_{\mathfrak{p}}}\left(R_{\mathfrak{p}} / I R_{\mathfrak{p}}, \operatorname{Hom}_{R}\left(R_{\mathfrak{p}}, M\right)\right)=$ 0 for any $0 \leq i<n$ and for any $\mathfrak{p} \in \operatorname{Cos}_{R} M \cap \mathrm{V}(I)-X$. Moreover, there exists $\mathfrak{q} \in \operatorname{Cos}_{R} M \cap \mathrm{V}(I)-X$, such that $\operatorname{Tor}_{n}^{R_{\mathfrak{q}}}\left(R_{\mathfrak{q}} / I R_{\mathfrak{q}}, \operatorname{Hom}_{R}\left(R_{\mathfrak{p}}, M\right)\right) \neq 0$. Hence $\operatorname{cograde}_{X}(I, M)=\inf \left\{\operatorname{cograde}_{R_{\mathfrak{p}}}\left(I R_{\mathfrak{p}}, \operatorname{Hom}_{R}\left(R_{\mathfrak{p}}, M\right)\right) \mid \mathfrak{p} \in \operatorname{Cos}_{R} M \cap \mathrm{V}(I)-X\right\}$.

Let $R$ be Noetherian ring, $I$ a ideal of $R, N$ a finitely generated $R$-module. If $I N \neq N$, then the $\operatorname{depth}_{R}(I, N)<\infty$. The dual question for Artinian module $M$ is to ask when $\operatorname{cograde}_{X}(I, M)<\infty$. We will give some sufficient conditions for finiteness of $\operatorname{cograde}_{X}(I, M)$.

Lemma 4.3. Let $R$ be a Noetherian topological ring, $M$ a strongly representable linearly compact $R$-module. Then the following conditions are equivalent:

(i). $\operatorname{Ann}_{R}\left(0:_{M} \mathfrak{p}\right)=\mathfrak{p}$ for any $\mathfrak{p} \in \mathrm{V}\left(\operatorname{Ann}_{R} M\right)$.

(ii). $\operatorname{Cos}_{R}\left(0:_{M} I\right)=\operatorname{Cos}_{R} M \cap \mathrm{V}(I)$ for any ideal $I$ of $R$. 
Proof. $(i) \Rightarrow(i i)$. Let $I \subseteq R$ be an ideal. By [3, Corollary 4.3], we have

$$
\mathrm{V}\left(\operatorname{Ann}_{R}\left(0:_{M} I\right)\right)=\operatorname{Cos}_{R}\left(0:_{M} I\right) \subseteq \operatorname{Cos}_{R} M .
$$

Thus $\operatorname{Cos}_{R}\left(0:_{M} I\right) \subseteq \operatorname{Cos}_{R} M \cap \mathrm{V}(I)$.

For an ideal $I$ of $R$, if $\operatorname{Cos}_{R} M \cap \mathrm{V}(I)=\emptyset$, there is nothing to prove. Suppose that $\operatorname{Cos}_{R} M \cap \mathrm{V}(I) \neq \emptyset$. Let $\mathfrak{p} \in \operatorname{Cos}_{R} M \cap \mathrm{V}(I)$. Since

$\operatorname{Cos}_{R}\left(0:_{M} \mathfrak{p}\right)=\mathrm{V}\left(\operatorname{Ann}_{R}\left(0:_{M} \mathfrak{p}\right)\right)$, and $\operatorname{Ann}_{R}\left(0:_{M} \mathfrak{p}\right)=\mathfrak{p}$ for any $\mathfrak{p} \in \mathrm{V}\left(\operatorname{Ann}_{R} M\right)$ we have $\mathfrak{p} \in \operatorname{Cos}_{R}\left(0:_{M} \mathfrak{p}\right)$. Notice that

$$
\operatorname{Hom}_{R}\left(R_{\mathfrak{p}}, 0:_{M} I\right) \supseteq\left(0: \operatorname{Hom}_{R}\left(R_{\mathfrak{p}}, M\right) \mathfrak{p} R_{\mathfrak{p}}\right)=\operatorname{Hom}_{R}\left(R_{\mathfrak{p}}, 0:_{M} \mathfrak{p}\right) \neq 0
$$

Hence $\operatorname{Cos}_{R} M \cap \mathrm{V}(I) \subseteq \operatorname{Cos}_{R}\left(0:_{M} I\right)$.

$(i i) \Rightarrow(i)$. Let $\mathfrak{p} \in \mathrm{V}\left(\operatorname{Ann}_{R} M\right)$. Then $\mathrm{V}\left(\operatorname{Ann}_{R}\left(0:_{M} \mathfrak{p}\right)\right)=\operatorname{Cos}_{R} M \cap \mathrm{V}(\mathfrak{p})=$ $\mathrm{V}(\mathfrak{p})$. Moreover, since $\mathfrak{p} \subseteq \operatorname{Ann}_{R}\left(0:_{M} \mathfrak{p}\right)$, we have $\operatorname{Ann}_{R}\left(0:_{M} \mathfrak{p}\right)=\mathfrak{p}$.

Proposition 4.4. Let $R$ be a Noetherian topological ring, $X$ a saturated subset of Spec $R$ and $I \subsetneq R$ a proper ideal of $R$. Let $M$ be a strongly representable linearly compact $R$-module such that $\operatorname{Ann}_{R}\left(0:_{M} \mathfrak{p}\right)=\mathfrak{p}$ for any $\mathfrak{p} \in \mathrm{V}\left(\operatorname{Ann}_{R} M\right)$. Then $\operatorname{cograde}_{X}(I, M)<\infty$ if and only if $\operatorname{Cos}_{R} M \cap \mathrm{V}(I) \nsubseteq X$.

Proof. $\Rightarrow$. If $\operatorname{Cos}_{R} M \cap \mathrm{V}(I) \subseteq X$, then $\operatorname{Cos}_{R}\left(\operatorname{Tor}_{i}^{R}(R / I, M)\right) \subseteq X$ for any $i \geq 0$, this contradicts to $\operatorname{cograde}_{X}(I, M)<\infty$. Hence $\operatorname{Cos}_{R} M \cap \mathrm{V}(I) \nsubseteq X X$.

$\Leftarrow$. For $\mathfrak{p} \in \operatorname{Cos}_{R} M \cap \mathrm{V}(I)-X$. Then by Lemma 4.3 we have

$$
\left(0: \operatorname{Hom}_{R}\left(R_{\mathfrak{p}}, M\right) I R_{\mathfrak{p}}\right)=\operatorname{Hom}_{R}\left(R_{\mathfrak{p}}, 0:_{M} I\right) \neq 0 .
$$

Let $\frac{x_{1}}{s_{1}}, \cdots, \frac{x_{r}}{s_{r}} \in I R_{\mathfrak{p}}$ be a $\operatorname{Hom}_{R}\left(R_{\mathfrak{p}}, M\right)$ co-regular sequence. Then

$$
0::_{\operatorname{Hom}_{R}\left(R_{\mathfrak{p}}, M\right)}\left(\frac{x_{1}}{s_{1}}\right) \supsetneq 0::_{\operatorname{Hom}_{R}\left(R_{\mathfrak{p}}, M\right)}\left(\frac{x_{1}}{s_{1}}, \frac{x_{2}}{s_{2}}\right) \supsetneq 0:_{\operatorname{Hom}_{R}\left(R_{\mathfrak{p}}, M\right)}\left(\frac{x_{1}}{s_{1}}, \frac{x_{2}}{s_{2}}, \cdots, \frac{x_{r}}{s_{r}}\right) .
$$

Otherwise, there must exist some integer $i, 1 \leq i \leq r$ such that $0: \operatorname{Hom}_{R}\left(R_{\mathfrak{p}}, M\right)$ $\left(\frac{x_{1}}{s_{1}}, \cdots, \frac{x_{i}}{s_{i}}\right)=0$. This contradicts to $0 \neq 0::_{\operatorname{Hom}_{R}\left(R_{\mathfrak{p}}, M\right)} I R_{\mathfrak{p}} \subseteq 0::_{\operatorname{Hom}_{R}\left(R_{\mathfrak{p}}, M\right)}$ $\left(\frac{x_{1}}{s_{1}}, \cdots, \frac{x_{i}}{s_{i}}\right)$. It follows that $\left(\frac{x_{1}}{s_{1}}\right) \subsetneq\left(\frac{x_{1}}{s_{1}}, \frac{x_{2}}{s_{2}}\right) \subsetneq \cdots \subsetneq\left(\frac{x_{1}}{s_{1}}, \frac{x_{2}}{s_{2}}, \cdots, \frac{x_{r}}{s_{r}}\right)$. Since $R_{\mathfrak{p}}$ is a Noetherian ring, therefore every $\operatorname{Hom}_{R}\left(R_{\mathfrak{p}}, M\right)$ co-regular sequence in $I R_{\mathfrak{p}}$ must be of finite length. Hence there exists an integer $n \geq 0$, such that

$$
\operatorname{Tor}_{n}^{R_{\mathfrak{p}}}\left(R_{\mathfrak{p}} / I R_{\mathfrak{p}}, \operatorname{Hom}_{R}\left(R_{\mathfrak{p}}, M\right)\right) \neq 0 \text {. }
$$

Thus $\operatorname{Cos}_{R}\left(\operatorname{Tor}_{n}^{R}(R / I, M)\right) \nsubseteq X$. Hence $\operatorname{cograde}_{X}(I, M)<\infty$.

Remark 4.5. By the proof of Proposition 4.4 we note that if $R$ is a Noetherian topological ring, and $M$ is a strongly representable linearly compact $R$-module such that $\operatorname{Ann}_{R}\left(0:_{M} \mathfrak{p}\right)=\mathfrak{p}$ for any $\mathfrak{p} \in \mathrm{V}\left(\operatorname{Ann}_{R} M\right)$, then $\operatorname{cograde}_{R_{\mathfrak{p}}} \operatorname{Hom}_{R}\left(R_{\mathfrak{p}}, M\right)<\infty$ for any $\mathfrak{p} \in \operatorname{Cos}_{R} M$.

N. T. Cuong, N. T. Dung and L. T. Nhan given an example to show that the equivalent conditions of Lemma 4.3 may not true for general Artinian modules ([5]). However, they also given some sufficient conditions for that condition.

Proposition 4.6. [5, Proposition 2.1] Let $(R, \mathfrak{m})$ be a Noetherian local ring, $M$ an Artinian $R$-module. If one of the following cases happens:

(1). $R$ is complete with respect to $\mathfrak{m}$-adic topology.

(1). $M$ contain a submodule which is isomorphic to the injective hull of $R / \mathfrak{m}$.

Then $\operatorname{Ann}_{R}(0: M \mathfrak{p})=\mathfrak{p}$ for any $\mathfrak{p} \in \mathrm{V}\left(\operatorname{Ann}_{R} M\right)$. 


\section{Vanishing Theorem of Dual Bass numbers}

Let $R$ be a Noetherian ring, $M$ an $R$-module. A minimal flat resolution of $M$ is an exact sequence of $R$-modules

$$
\cdots \stackrel{d_{i+1}}{\rightarrow} F_{i} \stackrel{d_{i}}{\rightarrow} F_{i-1} \stackrel{d_{i-1}}{\rightarrow} \cdots \stackrel{d_{1}}{\rightarrow} F_{0} \stackrel{d_{0}}{\rightarrow} M \rightarrow 0,
$$

such that for each $i \geq 0, F_{i}$ is a flat cover of $\operatorname{Im}\left(d_{i}\right)$. The minimal flat resolution of $M$ exists and uniquely determined up to isomorphism. Moreover, for any integer $n, \operatorname{fd}_{R} M \leq n$ if and only if $F_{k}=0$ for any $k>n$. In this case, $F_{i}$ is a flat cotorsion $R$-module for any $i>0$, but $F_{0}$ may not be cotorsion in general. E. Enochs [6] proved that $F_{i}$ is uniquely represented as a product $F_{i}=\prod_{\mathfrak{p} \in \text { Spec } R} T_{\mathfrak{p}}^{i}$, where $T_{\mathfrak{p}}^{i}$ is the completion of a free $R_{\mathfrak{p}}$-module with respect to the $\mathfrak{p} R_{\mathfrak{p}}$-adic topology. In [7, $\pi_{i}(\mathfrak{p}, M)$ is defined to be the cardinality of the base of a free $R_{\mathfrak{p}}$-module whose completion is $T_{\mathfrak{p}}^{i}$ for any $i>0$. On the other hand, $\pi_{0}(\mathfrak{p}, M)$ is defined similarly by using the pure injective envelope $P E\left(F_{0}\right)$ instead of $F_{0}$ itself. We call the $\pi_{i}(\mathfrak{p}, M)$ the $i$-th dual Bass number of $M$ with respect to $\mathfrak{p}$. E. Enochs and J. Z. Xu 7 , Theorem 2.2] showed that for any $R$-module $M$ over Noetherian ring $R$, there exists cotorsion $R$-module $E$ such that $\pi_{i}(\mathfrak{p}, M)=\pi_{i}(\mathfrak{p}, E)$ for any $i \geq 0$. The main results of [7] is the following: Let $R$ be a Noetherian ring, $M$ a cotorsion $R$-module. Then $\pi_{i}(\mathfrak{p}, M)=\operatorname{dim}_{k(\mathfrak{p})} \operatorname{Tor}_{i}^{R_{\mathfrak{p}}}\left(k(\mathfrak{p}), \operatorname{Hom}_{R}\left(R_{\mathfrak{p}}, M\right)\right)$, for any $i \geq 0$.

By the proof of [7, Theorem 2.2], we could get following Lemma.

Lemma 5.1. Let $R$ be a Noetherian ring, $\mathfrak{p}, \mathfrak{q} \in \operatorname{Spec} R$ with $\mathfrak{q} \subseteq \mathfrak{p}$, and $M a$ cotorsion $R$-module. Let the following exact sequence

$$
\stackrel{d_{i+1}}{\rightarrow} F_{i} \stackrel{d_{i}}{\rightarrow} F_{i-1} \stackrel{d_{i-1}}{\rightarrow} \cdots \stackrel{d_{1}}{\rightarrow} F_{0} \stackrel{d_{0}}{\rightarrow} M \rightarrow 0
$$

be a minimal flat resolution of $M$. Then

$$
\begin{gathered}
\cdots \rightarrow \operatorname{Hom}_{R}\left(R_{\mathfrak{p}}, F_{i+1}\right) \rightarrow \operatorname{Hom}_{R}\left(R_{\mathfrak{p}}, F_{i}\right) \rightarrow \operatorname{Hom}_{R}\left(R_{\mathfrak{p}}, F_{i-1}\right) \rightarrow \\
\quad \cdots \rightarrow \operatorname{Hom}_{R}\left(R_{\mathfrak{p}}, F_{1}\right) \rightarrow \operatorname{Hom}_{R}\left(R_{\mathfrak{p}}, F_{0}\right) \rightarrow \operatorname{Hom}_{R}\left(R_{\mathfrak{p}}, M\right) \rightarrow 0
\end{gathered}
$$

is a minimal flat resolution of $\operatorname{Hom}_{R}\left(R_{\mathfrak{p}}, M\right)$ as an $R_{\mathfrak{p}}$-module, and if $F_{i}=\prod_{\mathfrak{q} \in \operatorname{Spec} R} T_{\mathfrak{q}}^{i}$, then $\operatorname{Hom}_{R}\left(R_{\mathfrak{p}}, F_{i}\right) \cong \prod_{\substack{\mathfrak{q} \mathfrak{p} \mathfrak{q} \\ \mathfrak{q} \in \operatorname{Spec} R}} T_{\mathfrak{q}}^{i}$ is a flat cotorsion $R_{\mathfrak{p}}$-module. In other words, $\pi_{i}(\mathfrak{q}, M)=\pi_{i}\left(\mathfrak{q} R_{\mathfrak{p}}, \operatorname{Hom}_{R}\left(R_{\mathfrak{p}}, M\right)\right)$ for any $i \geq 0$.

Proposition 5.2. Let $R$ be a Noetherian ring, $M$ an Artinian $R$-module. Then for any $\mathfrak{p} \in \operatorname{Cos}_{R} M, \operatorname{cograde}_{R_{\mathfrak{p}}} \operatorname{Hom}_{R}\left(R_{\mathfrak{p}}, M\right)=\inf \left\{i \mid \pi_{i}(\mathfrak{p}, M)>0\right\}$.

Proof. Since any Artinian module is cotorsion (17, Theorem 2.8]). Then the formula $\pi_{i}(\mathfrak{p}, M)=\operatorname{dim}_{k(\mathfrak{p})} \operatorname{Tor}_{i}^{R_{\mathfrak{p}}}\left(k(\mathfrak{p}), \operatorname{Hom}_{R}\left(R_{\mathfrak{p}}, M\right)\right)$ holds for any Artinian module. Hence, this Proposition follows from Proposition 4.1.

Lemma 5.3. Let $R$ be a Noetherian ring, $F$ a flat cotorsion $R$-module. Then

$$
F \neq 0 \Leftrightarrow \operatorname{Cos}_{R} F \neq \emptyset \text {. }
$$

Proof. Suppose that $F \neq 0$. Assume that $F=\prod_{\mathfrak{p} \in \operatorname{Spec} R} T_{\mathfrak{p}}$, where $T_{\mathfrak{p}}$ is the completion of a free $R_{\mathfrak{p}}$-module with respect to the $\mathfrak{p} R_{\mathfrak{p}}$-adic topology. Then there exists $\mathfrak{p} \in \operatorname{Spec} R$ such that $T_{\mathfrak{p}} \neq 0$. Then we have $\operatorname{Hom}_{R}\left(R_{\mathfrak{p}}, T_{\mathfrak{p}}\right) \cong T_{\mathfrak{p}} \neq 0$. Hence $\operatorname{Hom}_{R}\left(R_{\mathfrak{p}}, F\right) \neq 0$, and thus $\operatorname{Cos}_{R} F \neq \emptyset$. Conversely, it is obvious. 
Proposition 5.4. Let $R$ be a Noetherian ring, $M$ a cotorsion $R$-module. Then:

(1). $\mathrm{fd}_{S^{-1} R} \operatorname{Hom}_{R}\left(S^{-1} R, M\right) \leq \mathrm{fd}_{R} M$ for any multiplicative set $S \subseteq R$.

(2). $\operatorname{fd}_{R} M=\sup \left\{\operatorname{fd}_{R_{\mathfrak{p}}} \operatorname{Hom}_{R}\left(R_{\mathfrak{p}}, M\right) \mid \mathfrak{p} \in \operatorname{Spec} R\right\}$

$=\sup \left\{\operatorname{fd}_{R_{\mathfrak{m}}} \operatorname{Hom}_{R}\left(R_{\mathfrak{m}}, M\right) \mid \mathfrak{m} \in \operatorname{Max} R\right\}$.

Proof. Let the following exact sequence of $R$-modules

$$
\cdots \rightarrow F_{i} \rightarrow F_{i-1} \rightarrow \cdots \rightarrow F_{0} \rightarrow M \rightarrow 0
$$

be a minimal flat resolution of $M$. Then by [7, Theorem 2.7], we have a minimal flat resolution of $S^{-1} R$-module $\operatorname{Hom}_{R}\left(S^{-1} R, M\right)$

$$
\begin{gathered}
\cdots \rightarrow \operatorname{Hom}_{R}\left(S^{-1} R, F_{i+1}\right) \rightarrow \operatorname{Hom}_{R}\left(S^{-1} R, F_{i}\right) \rightarrow \operatorname{Hom}_{R}\left(S^{-1} R, F_{i-1}\right) \rightarrow \\
\cdots \rightarrow \operatorname{Hom}_{R}\left(S^{-1} R, F_{1}\right) \rightarrow \operatorname{Hom}_{R}\left(S^{-1} R, F_{0}\right) \rightarrow \operatorname{Hom}_{R}\left(S^{-1} R, M\right) \rightarrow 0 .
\end{gathered}
$$

Then (1) follows. By Lemma 5.3. (2) also holds.

Proposition [5.4 is somehow dual to [1, Corollary 2.3].

Definition 5.5. A Noetherian ring $R$ is called a $U$ ring, if for any Artinian $R$ module $M, \operatorname{Ann}_{R}\left(0:_{M} \mathfrak{p}\right)=\mathfrak{p}$ for any $\mathfrak{p} \in \mathrm{V}\left(\operatorname{Ann}_{R} M\right)$.

Notice that any complete Noetherian local rings is a $U$ ring by [5 Proposition 2.1]. Let $M$ be an Artinian $R$-module over a $U$ ring. Then for any $\mathfrak{p} \in \operatorname{Cos}_{R} M$,

$$
\operatorname{cograde}_{R_{\mathfrak{p}}} \operatorname{Hom}_{R}\left(R_{\mathfrak{p}}, M\right)<\infty,
$$

because $\left(0:_{\left.\operatorname{Hom}_{R}\left(R_{\mathfrak{p}}, M\right)\right)} \mathfrak{p} R_{\mathfrak{p}}\right) \cong \operatorname{Hom}_{R}\left(R_{\mathfrak{p}}, 0:_{M} \mathfrak{p}\right) \neq 0$ and any co-regular sequence in Noetherian ring must be of finite length.

Theorem 5.6. Let $R$ be a $U$ ring, $\mathfrak{p}, \mathfrak{q} \in \operatorname{Spec} R$ such that $\mathfrak{q} \subset \mathfrak{p}, \operatorname{ht}(\mathfrak{p} / \mathfrak{q})=1$, and $M$ an Artinian $R$-module. Then $\pi_{i}(\mathfrak{q}, M) \neq 0 \Rightarrow \pi_{i+1}(\mathfrak{p}, M) \neq 0$.

Proof. For $x \in \mathfrak{p}-\mathfrak{q}$, from the short exact sequence of $R_{\mathfrak{p}}$-modules

$$
0 \rightarrow R_{\mathfrak{p}} / \mathfrak{q} R_{\mathfrak{p}} \stackrel{\frac{x}{1}}{\rightarrow} R_{\mathfrak{p}} / \mathfrak{q} R_{\mathfrak{p}} \rightarrow R_{\mathfrak{p}} /\left(\mathfrak{q} R_{\mathfrak{p}}, \frac{x}{1}\right) \rightarrow 0,
$$

we get the following long exact sequences of $R_{\mathfrak{p}}$-modules

$$
\begin{gathered}
\cdots \rightarrow \operatorname{Tor}_{i+1}^{R_{\mathfrak{p}}}\left(R_{\mathfrak{p}} /\left(\mathfrak{q} R_{\mathfrak{p}}, \frac{x}{1}\right), \operatorname{Hom}_{R}\left(R_{\mathfrak{p}}, M\right)\right) \rightarrow \operatorname{Tor}_{i}^{R_{\mathfrak{p}}}\left(R_{\mathfrak{p}} / \mathfrak{q} R_{\mathfrak{p}}, \operatorname{Hom}_{R}\left(R_{\mathfrak{p}}, M\right)\right) \stackrel{\frac{x}{1}}{\rightarrow} \\
\operatorname{Tor}_{i}^{R_{\mathfrak{p}}}\left(R_{\mathfrak{p}} / \mathfrak{q} R_{\mathfrak{p}}, \operatorname{Hom}_{R}\left(R_{\mathfrak{p}}, M\right)\right) \rightarrow \operatorname{Tor}_{i}^{R_{\mathfrak{p}}}\left(R_{\mathfrak{p}} /\left(\mathfrak{q} R_{\mathfrak{p}}, \frac{x}{1}\right), \operatorname{Hom}_{R}\left(R_{\mathfrak{p}}, M\right)\right) \rightarrow \cdots
\end{gathered}
$$

Since

$$
\begin{aligned}
\operatorname{Hom}_{R_{\mathfrak{p}}}\left(R_{\mathfrak{q}}, \operatorname{Tor}_{i}^{R_{\mathfrak{p}}}\left(R_{\mathfrak{p}} / \mathfrak{q} R_{\mathfrak{p}}, \operatorname{Hom}_{R}\left(R_{\mathfrak{p}}, M\right)\right)\right) & \cong \operatorname{Hom}_{R_{\mathfrak{p}}}\left(R_{\mathfrak{q}}, \operatorname{Hom}_{R}\left(R_{\mathfrak{p}}, \operatorname{Tor}_{i}^{R}(R / \mathfrak{q}, M)\right)\right) \\
& \cong \operatorname{Hom}_{R}\left(R_{\mathfrak{q}}, \operatorname{Tor}_{i}^{R}(R / \mathfrak{q}, M)\right) \\
& \cong \operatorname{Tor}_{i}^{R_{\mathfrak{q}}}\left(k(\mathfrak{q}), \operatorname{Hom}_{R}\left(R_{\mathfrak{q}}, M\right)\right) \neq 0,
\end{aligned}
$$

we have $\operatorname{Tor}_{i}^{R_{\mathfrak{p}}}\left(R_{\mathfrak{p}} / \mathfrak{q} R_{\mathfrak{p}}, \operatorname{Hom}_{R}\left(R_{\mathfrak{p}}, M\right)\right) \neq 0$. Thus $\mathfrak{p} \in \operatorname{Cos}_{R} \operatorname{Tor}_{i}^{R}(R / \mathfrak{q}, M)$. Notice that $\operatorname{Tor}_{i}^{R}(R / \mathfrak{q}, M)$ is an Artinian $R$-module, then

$$
\operatorname{Cos}_{R}\left(0: \operatorname{Tor}_{i}^{R}(R / \mathfrak{q}, M) x\right)=\operatorname{Cos}_{R} \operatorname{Tor}_{i}^{R}(R / \mathfrak{q}, M) \cap V((x)) .
$$

Since $x \in \mathfrak{p}$, we get $\mathfrak{p} \in \operatorname{Cos} R\left(0:_{\operatorname{Tor}_{i}^{R}(R / \mathfrak{q}, M)} x\right)$. Hence

$$
\begin{aligned}
\left(0::_{\operatorname{Tor}_{i} R_{\mathfrak{p}}}\left(R_{\mathfrak{p}} / \mathfrak{q} R_{\mathfrak{p}}, \operatorname{Hom}_{R}\left(R_{\mathfrak{p}}, M\right)\right) \frac{x}{1}\right) & \cong\left(0: \operatorname{Hom}_{R}\left(R_{\mathfrak{p}}, \operatorname{Tor}_{i}^{R}(R / \mathfrak{q}, M)\right) \frac{x}{1}\right) \\
& \cong \operatorname{Hom}_{R}\left(R_{\mathfrak{p}}, 0:_{\operatorname{Tor}_{i}^{R}(R / \mathfrak{q}, M)} x\right) \neq 0 .
\end{aligned}
$$


Thus, $\operatorname{Tor}_{i+1}^{R_{\mathfrak{p}}}\left(R_{\mathfrak{p}} /\left(\mathfrak{q} R_{\mathfrak{p}}, \frac{x}{1}\right), \operatorname{Hom}_{R}\left(R_{\mathfrak{p}}, M\right)\right) \neq 0$. Notice that $\operatorname{ht}(\mathfrak{p} / \mathfrak{q})=1$, therefore $R_{\mathfrak{p}} /\left(\mathfrak{q} R_{\mathfrak{p}}, \frac{x}{1}\right)$ is a finite length $R_{\mathfrak{p}}$-module. Let

$$
0=C_{0} \subsetneq C_{1} \subsetneq \cdots \subsetneq C_{n}=R_{\mathfrak{p}} /\left(\mathfrak{q} R_{\mathfrak{p}}, \frac{x}{1}\right)
$$

be a composition series of $R_{\mathfrak{p}} /\left(\mathfrak{q} R_{\mathfrak{p}}, \frac{x}{1}\right)$ as an $R_{\mathfrak{p}}$-module. Then $C_{j} / C_{j-1} \cong k(\mathfrak{p})$ for $j=1,2, \cdots, n$. By this we can draw a conclusion that

$$
\operatorname{Tor}_{i+1}^{R_{\mathfrak{p}}}\left(k(\mathfrak{p}), \operatorname{Hom}_{R}\left(R_{\mathfrak{p}}, M\right)\right) \neq 0 \text {. }
$$

Otherwise, by induction on the length of $R_{\mathfrak{p}} /\left(\mathfrak{q} R_{\mathfrak{p}}, \frac{x}{1}\right)$, we deduce that

$$
\operatorname{Tor}_{i+1}^{R_{\mathfrak{p}}}\left(R_{\mathfrak{p}} /\left(\mathfrak{q} R_{\mathfrak{p}}, \frac{x}{1}\right), \operatorname{Hom}_{R}\left(R_{\mathfrak{p}}, M\right)\right)=0
$$

This induces a contradiction.

Corollary 5.7. Let $R$ be a $U$ ring, $\mathfrak{p}, \mathfrak{q} \in \operatorname{Spec} R$ such that $\mathfrak{q} \subsetneq \mathfrak{p}, \operatorname{ht}(\mathfrak{p} / \mathfrak{q})=s$, and $M$ an Artinian $R$-module. Then $\pi_{i}(\mathfrak{q}, M) \neq 0 \Rightarrow \pi_{i+s}(\mathfrak{p}, M) \neq 0$.

Proof. Using induction on $\mathrm{ht}(\mathfrak{p} / \mathfrak{q})$. This Corollary follows from Theorem 5.6 ,

Corollary 5.8. Let $R$ be a $U$ ring, $\mathfrak{p} \in \operatorname{Spec} R, M$ an Artinian $R$-module. If there some $n \in \mathbb{N}$ such that $\pi_{i}(\mathfrak{p}, M)=0$ for any $i>n$, then $\operatorname{fd}_{R_{\mathfrak{p}}} \operatorname{Hom}_{R}\left(R_{\mathfrak{p}}, M\right) \leq n$.

Proof. If $\mathrm{fd}_{R_{\mathfrak{p}}} \operatorname{Hom}_{R}\left(R_{\mathfrak{p}}, M\right)>n$, then by Lemma 5.1 there exists $\mathfrak{q} \subseteq \mathfrak{p}, \mathfrak{q} \in$ Spec $R$, such that $\pi_{n+1}(\mathfrak{q}, M)>0$. Then we get $\pi_{\mathrm{ht}(\mathfrak{p} / \mathfrak{q})+n+1}(\mathfrak{p}, M)>0$ by Theorem 5.6. This contradicts to the assumption. Thus $\operatorname{fd}_{R_{\mathfrak{p}}} \operatorname{Hom}_{R}\left(R_{\mathfrak{p}}, M\right) \leq n$.

To obtain the main result of this section, we first prove the following Proposition.

Proposition 5.9. Let $R$ be a Noetherian topological ring, $M$ a strongly representable linearly compact $R$-module. If $\operatorname{cograde}_{R_{\mathfrak{p}}} \operatorname{Hom}_{R}\left(R_{\mathfrak{p}}, M\right)<\infty$, then

$$
\operatorname{cograde}_{R_{\mathfrak{p}}} \operatorname{Hom}_{R}\left(R_{\mathfrak{p}}, M\right) \leq \operatorname{Cdim}_{R_{\mathfrak{p}}} \operatorname{Hom}_{R}\left(R_{\mathfrak{p}}, M\right) .
$$

Proof. Let $n=\operatorname{cograde}_{R_{\mathfrak{p}}} \operatorname{Hom}_{R}\left(R_{\mathfrak{p}}, M\right)$. We use induction on $n$.

For the case $n=0$, this Proposition is obviously true.

Assume that $n>1$, and this Proposition holds for $n-1$. Let $\frac{x}{s} \in \mathfrak{p} R_{\mathfrak{p}}$ be a $\operatorname{Hom}_{R}\left(R_{\mathfrak{p}}, M\right)$ co-regular element. Then $\frac{x}{s} \in R_{\mathfrak{p}}-\bigcup_{\mathfrak{q} \in \operatorname{Att}_{R} M, \mathfrak{q} \subseteq \mathfrak{p}} \mathfrak{q} R_{\mathfrak{p}}$, and

$$
\operatorname{cograde}_{R_{\mathfrak{p}}}\left(0:_{\operatorname{Hom}_{R}\left(R_{\mathfrak{p}}, M\right)} \frac{x}{s}\right)=\operatorname{cograde}_{R_{\mathfrak{p}}} \operatorname{Hom}_{R}\left(R_{\mathfrak{p}},\left(0:_{M} x\right)\right)=n-1 .
$$

Hence $x \in R-\bigcup_{\mathfrak{q} \in \operatorname{Att}_{R} M, \mathfrak{q} \subseteq \mathfrak{p}} \mathfrak{q}$, and by induction hypothesis we get

$$
\operatorname{cograde}_{R_{\mathfrak{p}}} \operatorname{Hom}_{R}\left(R_{\mathfrak{p}}, 0:_{M} x\right) \leq \operatorname{Cdim}_{R_{\mathfrak{p}}} \operatorname{Hom}_{R}\left(R_{\mathfrak{p}}, 0:_{M} x\right) .
$$

Since

$$
\begin{aligned}
\operatorname{Cdim}_{R_{\mathfrak{p}}} \operatorname{Hom}_{R}\left(R_{\mathfrak{p}}, 0:_{M} x\right) & =\sup \left\{\operatorname{ht}(\mathfrak{p} / \mathfrak{q}) \mid \mathfrak{q} \in \operatorname{Cos}_{R}\left(0:_{M} x\right), \mathfrak{q} \subseteq \mathfrak{p}\right\} \\
& \leq \operatorname{ht}\left(\mathfrak{p} /\left(\operatorname{Ann}_{R} M, x\right)\right) \\
& =\max \left\{\operatorname{ht}(\mathfrak{p} /(\mathfrak{q}, x)) \mid \mathfrak{q} \in \operatorname{Att}_{R} M, \mathfrak{q} \subseteq \mathfrak{p}\right\} \\
& =\max \left\{\operatorname{ht}(\mathfrak{p} / \mathfrak{q})-1 \mid \mathfrak{q} \in \operatorname{Att}_{R} M, \mathfrak{q} \subseteq \mathfrak{p}\right\} \\
& =\operatorname{Cdim}_{R_{\mathfrak{p}}} \operatorname{Hom}_{R}\left(R_{\mathfrak{p}}, M\right)-1,
\end{aligned}
$$

we have $\operatorname{cograde}_{R_{\mathfrak{p}}} \operatorname{Hom}_{R}\left(R_{\mathfrak{p}}, M\right) \leq \operatorname{Cdim}_{R_{\mathfrak{p}}} \operatorname{Hom}_{R}\left(R_{\mathfrak{p}}, M\right)$. 
The following Theorem is the main result of this paper. We characterize the infimum and supremum of the index of non-vanishing dual Bass numbers of Artinian modules, and study the relations among cograde,co-dimension and flat dimension of co-localization of Artinian modules.

Theorem 5.10. Let $R$ be a $U$ ring, $M$ an Artinian $R$-module, $\mathfrak{p} \in \operatorname{Cos}_{R} M$. Then:

(1). If $\pi_{i}(\mathfrak{p}, M)>0$, then $\operatorname{cograde}_{R_{\mathfrak{p}}} \operatorname{Hom}_{R}\left(R_{\mathfrak{p}}, M\right) \leq i \leq \mathrm{fd}_{R_{\mathfrak{p}}} \operatorname{Hom}_{R}\left(R_{\mathfrak{p}}, M\right)$. If $\operatorname{cograde}_{R_{\mathfrak{p}}} \operatorname{Hom}_{R}\left(R_{\mathfrak{p}}, M\right)=s$, then $\pi_{s}(\mathfrak{p}, M)>0 .(R$ is a Noetherian ring, not necessarily a $U$ ring, and $\mathrm{fd}_{R_{\mathfrak{p}}} \operatorname{Hom}_{R}\left(R_{\mathfrak{p}}, M\right)$ is possibly infinite).

(2). If $\mathrm{fd}_{R_{\mathfrak{p}}} \operatorname{Hom}_{R}\left(R_{\mathfrak{p}}, M\right)=t<\infty, \mathfrak{p}, \mathfrak{q} \in \operatorname{Spec} R$ such that $\mathfrak{q} \subseteq \mathfrak{p}$, then

$$
\pi_{t}(\mathfrak{q}, M)>0 \Leftrightarrow \mathfrak{q}=\mathfrak{p} .
$$

(3). If $\operatorname{fd}_{R_{\mathfrak{p}}} \operatorname{Hom}_{R}\left(R_{\mathfrak{p}}, M\right)=\infty$. Then for any $n \in \mathbb{N}$, there exists $i \geq n$ such that $\pi_{i}(\mathfrak{p}, M)>0$.

(4). $\operatorname{cograde}_{R_{\mathfrak{p}}} \operatorname{Hom}_{R}\left(R_{\mathfrak{p}}, M\right) \leq \operatorname{Cdim}_{R_{\mathfrak{p}}} \operatorname{Hom}_{R}\left(R_{\mathfrak{p}}, M\right) \leq \operatorname{fd}_{R_{\mathfrak{p}}} \operatorname{Hom}_{R}\left(R_{\mathfrak{p}}, M\right)$.

Proof. (1). By Proposition 5.2, we only need to show

$$
\pi_{i}(\mathfrak{p}, M)>0 \Rightarrow i \leq \mathrm{fd}_{R_{\mathfrak{p}}} \operatorname{Hom}_{R}\left(R_{\mathfrak{p}}, M\right) .
$$

For the case $\operatorname{fd}_{R_{\mathfrak{p}}} \operatorname{Hom}_{R}\left(R_{\mathfrak{p}}, M\right)=\infty$, there is nothing to prove. Suppose that $\operatorname{fd}_{R_{\mathfrak{p}}} \operatorname{Hom}_{R}\left(R_{\mathfrak{p}}, M\right)=r<\infty$. If $i>r$ such that $\pi_{i}(\mathfrak{p}, M)>0$, then by Lemma 5.1 we get $\pi_{i}(\mathfrak{p}, M)=\pi_{i}\left(\mathfrak{p} R_{\mathfrak{p}}, \operatorname{Hom}_{R}\left(R_{\mathfrak{p}}, M\right)\right)$. Thus $\operatorname{fd}_{R_{\mathfrak{p}}} \operatorname{Hom}_{R}\left(R_{\mathfrak{p}}, M\right) \geq i$. This induces a contradiction. Hence $i \leq \mathrm{fd}_{R_{\mathfrak{p}}} \operatorname{Hom}_{R}\left(R_{\mathfrak{p}}, M\right)$.

(2). Since $\operatorname{fd}_{R_{\mathfrak{p}}} \operatorname{Hom}_{R}\left(R_{\mathfrak{p}}, M\right)=t<\infty$, then by Lemma 5.1 there must exist $\mathfrak{q} \subseteq \mathfrak{p}, \mathfrak{q} \in \operatorname{Spec} R$ such that $\pi_{t}(\mathfrak{q}, M)>0$. Suppose that $\mathfrak{q} \subsetneq \mathfrak{p}$. Then by Corollary 5.7 we have $\pi_{t+\mathrm{ht}(\mathfrak{p} / \mathfrak{q})}(\mathfrak{p}, M)=\pi_{t+\mathrm{ht}(\mathfrak{p} / \mathfrak{q})}\left(\mathfrak{p} R_{\mathfrak{p}}, \operatorname{Hom}_{R}\left(R_{\mathfrak{p}}, M\right)\right) \neq 0$. This contradict to $\operatorname{fd}_{R_{\mathfrak{p}}} \operatorname{Hom}_{R}\left(R_{\mathfrak{p}}, M\right)=t$. Hence $\mathfrak{q}=\mathfrak{p}$. It is similar to prove the other side.

(3). This conclusion is followed by Corollary 5.8

(4). By Proposition 5.9 we only need to show

$$
\operatorname{Cdim}_{R_{\mathfrak{p}}} \operatorname{Hom}_{R}\left(R_{\mathfrak{p}}, M\right) \leq \mathrm{fd}_{R_{\mathfrak{p}}} \operatorname{Hom}_{R}\left(R_{\mathfrak{p}}, M\right) .
$$

Let $\mathfrak{q}$ be a minimal element of $\operatorname{Cos}_{R} M$ with $\mathfrak{q} \subseteq \mathfrak{p}$, by [3, Theorem 4.5], we get $\mathfrak{q} \in \operatorname{Att}_{R}\left(R / \mathfrak{q} \otimes_{R} M\right) \subseteq \operatorname{Cos}_{R}\left(R / \mathfrak{q} \otimes_{R} M\right)$. Since

$\pi_{0}(\mathfrak{q}, M)=\operatorname{dim}_{k(\mathfrak{q})} k(\mathfrak{q}) \otimes_{R_{\mathfrak{q}}} \operatorname{Hom}_{R}\left(R_{\mathfrak{q}}, M\right)=\operatorname{dim}_{k(\mathfrak{q})} \operatorname{Hom}_{R}\left(R_{\mathfrak{q}}, R / \mathfrak{q} \otimes_{R} M\right) \neq 0$

By Corollary 5.7, we get $\pi_{\mathrm{ht}(\mathfrak{p} / \mathfrak{q})}(\mathfrak{p}, M)=\pi_{\mathrm{ht}(\mathfrak{p} / \mathfrak{q})}\left(\mathfrak{p} R_{\mathfrak{p}}, \operatorname{Hom}_{R}\left(R_{\mathfrak{p}}, M\right)\right) \neq 0$. On the other hand, $\operatorname{Cdim}_{R_{\mathfrak{p}}} \operatorname{Hom}_{R}\left(R_{\mathfrak{p}}, M\right)=\max \left\{\operatorname{ht}(\mathfrak{p} / \mathfrak{q}) \mid \mathfrak{q} \in \operatorname{Cos}_{R} M, \mathfrak{q} \subseteq \mathfrak{p}\right\}$. Hence, $\operatorname{Cdim}_{R_{\mathfrak{p}}} \operatorname{Hom}_{R}\left(R_{\mathfrak{p}}, M\right) \leq \mathrm{fd}_{R_{\mathfrak{p}}} \operatorname{Hom}_{R}\left(R_{\mathfrak{p}}, M\right)$.

Corollary 5.11. Let $R$ be a $U$ ring, $M$ an Artin $R$-module. Then:

(1). $\operatorname{Cdim}_{R} M \leq \mathrm{fd}_{R} M$.

(2). If $\operatorname{fd}_{R} M=r<\infty$, and $\pi_{r}(\mathfrak{p}, M) \neq 0$ for some $\mathfrak{p} \in \operatorname{Spec} R$, then $\mathfrak{p} \in \operatorname{Max} R$.

Proof. (1). Since $\operatorname{Cdim}_{R} M=\sup \left\{\operatorname{Cdim}_{R_{\mathfrak{m}}} \operatorname{Hom}_{R}\left(R_{\mathfrak{m}}, M\right) \mid \mathfrak{m} \in \operatorname{Max} R\right\}$, by Proposition 5.4 (2) and Theorem 5.10 (4) we have $\operatorname{Cdim}_{R} M \leq \operatorname{fd}_{R} M$.

(2). If $\pi_{r}(\mathfrak{p}, M) \neq 0$, and $\mathfrak{p}$ is not a maximal ideal. It follows that there exists a maximal ideal $\mathfrak{m}$ such that $\mathfrak{p} \subsetneq \mathfrak{m}$, then by Corollary $5.7 \pi_{r+\mathrm{ht}(\mathfrak{m} / \mathfrak{p})}(\mathfrak{p}, M) \neq 0$. This contradict to $\operatorname{fd}_{R} M=r$. Hence, $\mathfrak{p}$ must be a maximal ideal. 


\section{REFERENCES}

[1] H. Bass: On the ubiquity of Gorenstein rings, Math. Z. 82 (1963), 8-28.

[2] N. T. Cuong and T. T. Nam: On the co-localization, co-support and co-associated primes of local homology modules, Vietnam J. Maths. 29 (2001), 359-368.

[3] N. T. Cuong and L. T. Nhan: On representable linearly compact modules, Proc. Amer. Math. Soc. 130 (2002), 1927-1936.

[4] N. T. Cuong and L. T. Nhan: On the Noetherian dimension of Artinian modules, Vietnam J. Maths. 30 (2002), 121-130.

[5] N. T. Cuong, N. T. Dung and L. T. Nhan: Top local cohomology and the catenaricity of the unmixed support of a finitely generated module, Comm. Algebra. 35 (2007), 1691-1701.

[6] E. Enochs: Flat covers and flat cotorsion modules, Proc. Amer. Math. Soc. 92 (1984), 179184.

[7] E. Enochs and J. Z. Xu: On invariants dual to the Bass numbers, Proc. Amer. Math. Soc. 125 (1997), 951-960.

[8] E. Enochs and O. M. Jenda: Relative Homological Algebra. de Gruyter Expositions in Mathematics. 30 (2000).

[9] D. Kirby: Dimension and length of Artinian modules, Quart. J. Math. Oxford. 41 (1990), 419-429.

[10] L. Melkersson and P. Schenzel: The co-localization of an Artinian module, Proc. Edinburgh Math. Soc. 38 (1995), 121-131.

[11] R. N. Roberts: Krull dimension for Artinian modules over quasi-local commutative rings, Quart. J. Math. Oxford. 26 (1975), 269-273.

[12] Z. Tang and H. Zakeri: Co-Cohen-Macaulay modules and modules of generalized fractions, Comm. Algebra. 22 (1994), 2173-2204.

[13] Z. Tang: Co-Cohen-Macaulay modules and multiplicities for Arinian modules, J. Suzhou Univ. (natural science), 12 (1996), 15-26.

[14] J. Z. Xu: Minimal injective and flat resolutions of modules over Gorenstein rings, J. Algebra. 175 (1995), 451-477.

Department of Mathematics, Tongui University, Shanghai, P. R. China, School of Mathematical Sciences, Fudan University, Shanghai, P. R. China

E-mail address: LG.Lee@amss.ac.cn 